Brief article

\title{
Perceptual wholes can reduce the conscious accessibility of their parts
}

\author{
Ervin Poljac*, Lee de-Wit, Johan Wagemans \\ Laboratory of Experimental Psychology, University of Leuven (K.U. Leuven), Belgium
}

\section{A R T I C L E I N F O}

\section{Article history:}

Received 12 August 2011

Revised 11 January 2012

Accepted 11 January 2012

Available online 3 February 2012

\section{Keywords:}

Visual perception

Perceptual organization

Gestalt formation

Object recognition

Grouping

\begin{abstract}
A B S T R A C T
Humans can rapidly extract object and category information from an image despite surprising limitations in detecting changes to the individual parts of that image. In this article we provide evidence that the construction of a perceptual whole, or Gestalt, reduces awareness of changes to the parts of this object. This result suggests that the rapid extraction of a perceptual Gestalt, and the inaccessibility of the parts that make up that Gestalt, may in fact reflect two sides of the same coin whereby human vision provides only the most useful level of abstraction to conscious awareness.
\end{abstract}

(c) 2012 Elsevier B.V. All rights reserved.

\section{Introduction}

The human visual system provides rapid access to the gist of a given image (Thorpe, Fize, \& Marlot, 1996) whilst at the same time providing sometimes surprisingly limited access to the individual parts that make up this interpretation (Rensink, O'Regan, \& Clark, 1997). We provide evidence that these characteristics of human vision (rapid access to object or class-level information, and limited access to their parts), may reflect a common mechanism by which the human visual system seeks to provide only the most critical visual information to further stages of processing in the human brain.

To understand why human vision might employ such a strategy one needs to consider the hierarchical and parallel nature of visual information processing, in which different visual features activate neurons with different receptive field sizes, in different visual pathways. This distributed form of information processing leads to specific computational challenges (Treisman, 1996), and surprising behavioral consequences (Treisman \& Gelade, 1980), in

\footnotetext{
* Corresponding author. Address: Laboratory of Experimental Psychology, University of Leuven (K.U. Leuven), Tiensestraat 102, Bus 3711, BE3000 Leuven, Belgium. Tel.: +32 16326 143; fax: +32 16326099 .

E-mail address: Ervin.Poljac@psy.kuleuven.be (E. Poljac).
}

combining different aspects of visual information processing. Indeed, it is far from straightforward how this hierarchical and parallel architecture can sit in accordance with our unified conscious perception of the world.

One step in resolving this discrepancy between the complexity of the architecture of the visual system and the unity of conscious experience may lie in the fact that whilst information might be processed at many stages, these stages do not have equal access in determining the contents conscious experience. This fact is illustrated by Williams, Dang, and Kanwisher (2007) who found that although shape information is apparent in the pattern of activation seen at both higher (LOC) and lower (V1) levels of processing, only the patterns of activation at higher stages of the system can predict conscious shape judgments. Indeed, this differential access of representations at different levels of visual processing is evident in numerous domains of visual processing, from color and brightness constancy to binocular rivalry (Fang \& He, 2005).

The closer association between information processing at higher stages of the visual system and conscious experience is consistent with Hochstein and Ahissar (2002) assumption that the content of conscious experience is automatically dictated by higher level stages of visual processing, which are associated with the representation of more abstract and meaningful interpretations. 
Furthermore, Hochstein and Ahissar assume that the details from which a particular visual abstraction, or interpretation, is constructed are not immediately, or automatically, accessible to conscious awareness.

This article provides data that extends this position by demonstrating that the ability to form a more abstract representation of a given visual input (in terms of a particular object) does not simply mean that the details of this stimulus are not immediately or automatically accessible to conscious perception, but that these details become fundamentally less accessible. The idea that 'the whole' might decrease access to the parts, as tested in this article, was inspired by a visual illusion reported by Suchow and Alvarez (2011). ${ }^{1}$ In this illusion the authors found that the ability of participants to perceive the color changes in a ring of 100 dots was reduced when these dots were set in motion. This 'silencing' is explained in terms of the inability of single cells with small receptive fields to sample data over time regarding the color change in any individual dot. Whilst not entirely ruling out a potential role for 'motion silencing', the current results show that something in addition is occurring, such that a more psychological process results in the reduced accessibility to the changing parts of visual input when these can be interpreted as an object.

This possibility is tested in the current article by measuring participants' ability to detect color changes to the elements of biological figures. Biological motion has a number of characteristics that make it perfectly suited to test for the influence of a perceptual whole on the accessibility of its constituent parts. Firstly, biological motion of point-light walkers is a prototypical case of a complex hierarchical stimulus, defined by multiple elements, each having their own spatiotemporally defined trajectories, which is organized quickly and efficiently by the visual system in a hierarchical configuration in which the motions of the local elements (e.g., wrists and ankles) are coded relative to a more global, structural description. Johansson (1973) developed a coding theory in which this hierarchical representation played an important role, a framework that has been used to explain other multielement stimuli with varying spatiotemporal profiles (e.g., Cutting \& Proffitt, 1982; Restle, 1979). Secondly, more recent work has shown that despite the apparent complexity of biological motion perception, the perceptual Gestalt is constructed automatically by the visual system (Thornton \& Vuong, 2004) and that the construction of the perceptual whole enables the visual system to represent the relationship between the parts of this stimulus more efficiently (Tadin, Lappin, Blake, \& Grossman, 2002). Third, and most critically, the perception of a the whole Gestalt can be disrupted in a perfectly controlled manner that leaves the bottom up nature of the stimulus identical, via inverting that stimulus (Pavlova \& Sokolov, 2000; Saygin, Driver, \& de Sa, 2008; Sumi, 1984). If the perceptual accessibility of parts is reduced for an upright relative to an inverted biological figure, then this provides very direct evidence that the perception of a whole influences the conscious accessibility to the parts making up that whole.

\footnotetext{
1 Recently voted 'Best Illusion of the Year'.
}

\section{Method}

\subsection{Participants}

Ten participants (six female) from the undergraduate psychology program at the University of Leuven conducted the experiment for course credit. They all had normal or corrected-to-normal vision and passed the Ishihara colorblindness test. The study was conducted in accordance with the ethical standards laid down in the 1964 Declaration of Helsinki and was approved by the K.U. Leuven Ethics Committee. All participants gave their written informed consent.

\subsection{Stimuli}

The experimental stimuli were generated using Matlab (Mathworks Inc.) and Psychophysics toolbox extensions (Brainard, 1997; Pelli, 1997). The positions of dots (small colored circles of about $.3^{\circ}$ ) that defined the stimulus were derived from the joint positions of a human figure walking on a treadmill, sequences adopted from (Dekeyser, Verfaillie, \& Vanrie, 2002; Vanrie \& Verfaillie, 2004) based on motion capture data (Qualisys MacReflex). Extra dots were added to the figure between the joints, in total 70 dots (Fig. 1a, Video S1), resulting in a 'confetti walker'. In addition to this intact, upright representation that provided a compelling impression of a walking human figure, an inverted version was created by rotating the whole image by $180^{\circ}$, while all other stimulus characteristics were kept the same (Fig. 1b, Video S2). Consistent with previous reports, as a consequence of this inversion participants perceived a more disorganized pattern of dots. Finally, a phase-scrambled figure was created by randomly assigning the initial frame of each separate dot within a walking cycle (Fig. 1c, Video S3). While the number of dots and their characteristics remained the same as in the intact figure, there was no clear percept of a figure here, but rather a collection of dots without a well-defined shape.

\subsection{Experiment 1}

Experiment 1 used motionless displays, snapshots of postures from the walking cycle. In this case we chose postures with large lateral extension (the distance between opposite ankles) to facilitate the perception of a human figure. Participants were seated approximately $57 \mathrm{~cm}$ from the screen (Dell 19" CRT monitor) that displayed two figures in each trial, approximately $3^{\circ}$ eccentrically to the left and right of the central fixation dot. On one side, a comparison phase-scrambled figure was presented, on the other an intact upright or inverted human figure, or a phasescrambled figure. Initially each dot was individually assigned a particular color. This color then changed continuously moving through a color spectrum defined in the HSV color space, whereby hue was changing and saturation and value were kept at maximum (100\%). The rate at which this color changed was different for the dots making up the figure on the left and on the right. We asked three participants to adjust (decrease or increase) the rate of change 

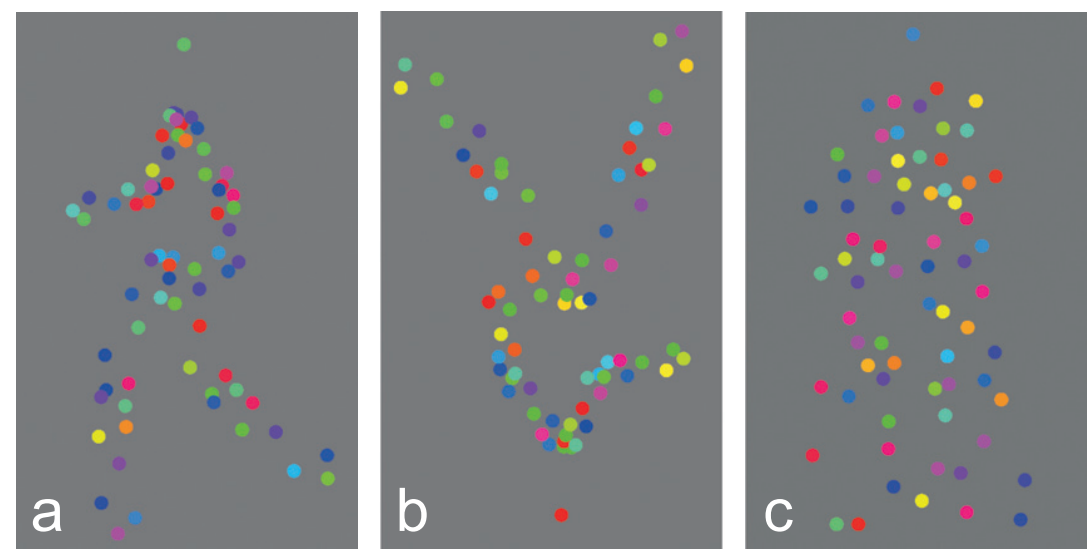

Fig. 1. Upright (a), inverted (b) and phase-scrambled (c) figure.

for one figure until it matched the rate of change in the comparison figure using the down and up arrow keys. Participants were instructed to compare all of the dots on one side with all of the dots on the other, while they looked at the fixation dot. Trials with upright and inverted stimuli were randomized but had an equal number of presentations, 160 in total. There was no time constraint.

\subsubsection{Results experiment 1}

Participants matched the rate of color change of one figure to apparent rate of color change in the other figure. We observed a clear inversion effect (see Fig. 2): The rate of color change in the upright stimulus is perceived as less than in the inverted figure (across subjects: $F=24.58$, $p=0.03$ ), an effect significant for each participant $(F=9.08, p=0.007 ; F=6.69, p=0.018 ; F=5.77, p=0.027$, respectively). All three participants were able to correctly match the color change rate in the two motionless phase-scrambled stimuli (difference in adjusted color change rate not significant $t(119)<1)$.

\subsection{Experiment 2}

Next, seven participants were asked to adjust the perceived rate of color change in presentations similar as above, but this time dynamic (intact upright or inverted,

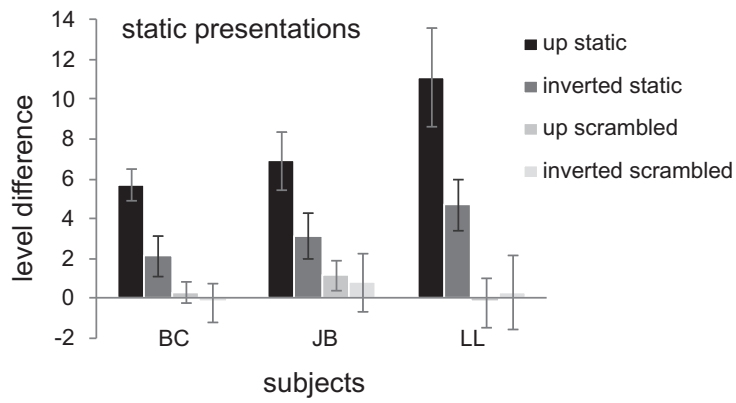

Fig. 2. Motionless presentations of upright and inverted figures for three participants. Error bars denote 1SE. The unit of the level difference axis represents $10.8 \% \mathrm{~s}$ around the color wheel. or phase-scrambled) and motionless figures were used. In each trial, on one side of the central fixation dot a phasescrambled figure was presented, on the other intact upright or inverted human figure (in equal number of trials). Figures on both sides could be either motionless or dynamic. These different combinations were randomly presented and appeared in equal number of trials. Again, participants were instructed to pay attention to all of the dots while they looked at the central fixation dot.

\subsubsection{Results experiment 2}

When adjusted to motionless images, the apparent rate of color change in a dynamic stimulus always appeared higher $(F=49.9$; $p<.001)$ (replication of Suchow and Alvarez's (2011) finding). More important for the current study is the difference between the color change rate appearance of the upright and inverted stimuli (Fig. 3). There was a clear effect of inversion in both motionless and dynamic stimuli $(F=19.53 ; p=.007)$. More specifically, the adjusted rate of change for upright stimuli was higher than for inverted ones. There was no interaction $(F=2.45 ; p=.2)$.

\section{Discussion}

The recognition of meaningful objects is something the brain performs with unparalleled speed and accuracy

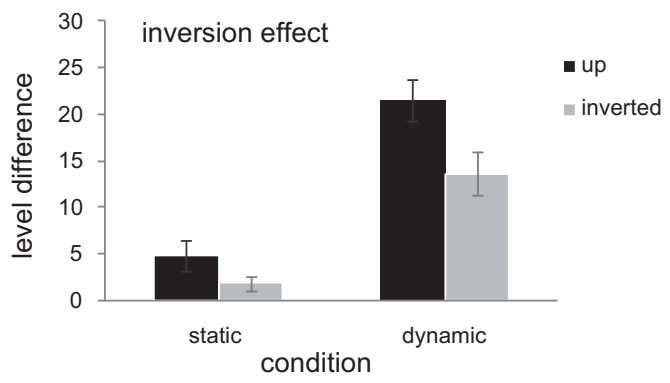

Fig. 3. Inversion effect observed for motionless and dynamic presentations. Error bars denote 1SE. 
(Thorpe et al., 1996). Furthermore objects can provide an efficient reference frame when judging the relationship between individual parts (Tadin et al., 2002). In this article, however, a cost of object-hood is found, in terms of the reduced accessibility to the changing features of the parts, when these parts can be more readily integrated into the perception of a whole object.

This potentially paradoxical picture whereby objects are rapidly extracted, and provide a useful reference frame, but at the same time lead to the reduced accessibility of the parts making up that object was predicted in terms of Hochstein and Ahissar's reversed hierarchy. This model postulates that the information upon which conscious perception is based is by default extracted from the most abstract (and therefore global and meaningful) stage of visual information processing, and that feedback mechanisms are required to go back and extract the details from which this abstraction is made. This led to the prediction that the parts making up a stimulus that can be more readily represented at a higher level of abstraction would be less accessible. Critically, this reduced accessibility was clear for our confetti walker even while participants could view the stimulus for an unconstrained amount of time.

This result suggests that whilst perceptual Gestalts might provide a more efficient reference frame within which to compare parts (Cutting \& Proffitt, 1982; Tadin et al., 2002), this efficiency comes at a cost in terms of the accessibility to those parts. The current result is consistent with studies on visual short-term memory for faces (Wilford \& Wells, 2010) and objects (Archambault, O'Donnell, \& Schyns, 1999) where the level at which a stimulus can be extracted (manipulated via inversion for faces, and previous categorization training for objects) reduces one's ability to remember details about the location or identity of parts. The current research builds on these findings by demonstrating that this reduced accessibility to parts affects not only one's short term memory, but that one's direct visual awareness of current sensory input is influenced by the construction of a perceptual Gestalt. The current finding also builds on Suchow and Alvarez (2011) results by demonstrating that something other than 'motion silencing' can reduce the perceptual accessibility to color change. Suchow and Alvarez suggest that the reduced accessibility to individual parts in their moving stimuli was caused by the movement of that stimulus across different receptive fields, something that would render the calculation of the signal change in a single neuron with a fixed receptive field impossible. Whilst not putting us in a position to rule out this sensory explanation per se, the current results suggest that the common fate of the dots moving together could lead to a perceptual grouping that itself renders the parts less accessible. Indeed independent of a process of sensory, motion silencing, our results provide clear evidence that even for motionless dots a more mid-level process of perceptual organization can reduced the accessibility of parts.

The notion that perceptual Gestalts can render their parts inaccessible is implicit in the study of embedded figures (Gottschaldt, 1926). Indeed, these figures provide a compelling demonstration of the idea that a perceptual part (such as a simple shape or an oriented line) can be very hard to identify when presented as part of a larger figure. Embedded figures however do not provide clear evidence for a psychological process by which parts become less accessible, simply because they contain many different local features, that will affect bottom up stimulus processing (such as end-stopping sensitive neurons) that could in principle explain the reduced accessibility to parts. In this regard the influence of inversion on biological motion perception offers an ideally controlled window into the role of the construction of Gestalts on the nature of our visual awareness.

The question of what determines the nature of conscious experience was a classical question in Gestalt psychology often framed simply as the question: "why do things look as they do' (Koffka, 1935). In this regard it has long been recognized that, in terms of conscious experience, the whole can look very different to the sum its parts. The current result helps to clarify why our perception of a whole might seem so dramatically different from a collection of parts. In order to streamline the transfer of information from a complicated visual hierarchy to conscious perception, the visual system does not simply construct wholes that reflect a non-linear combination of parts; rather the perception of a whole can silence those parts from conscious awareness. Indeed reflecting more generally, the construction of a perceptual Gestalt in the visual system is merely one instance in which the brain creates an abstract whole on the basis of multiple parts. This finding therefore provides a starting point in testing a more general computational principle wherein the formation of a more abstract representation may lead to a reduction in the conscious accessibility of the information from which that abstraction is formed.

\section{Acknowledgments}

This work was supported by the Methusalem Program by the Flemish Government (METH/08/02), awarded to Johan Wagemans. We would like to thank Frank Amand for technical support. Ervin Poljac and Lee de-Wit contributed equally to this work.

\section{Appendix A. Supplementary data}

Supplementary data associated with this article can be found, in the online version, at doi:10.1016/ j.cognition.2012.01.001.

\section{References}

Archambault, A., O’Donnell, C., \& Schyns, P. (1999). Blind to object changes: When learning the same object at different levels of categorization modifies its perception. Psychological Science, 10(3), 249-255.

Brainard, D. (1997). The psychophysics toolbox. Spatial Vision, 10(4), 433-436.

Cutting, J. E., \& Proffitt, D. R. (1982). The minimum principle and the perception of absolute, common, and relative motions. Cognitive Psychology, 14(2), 211-246.

Dekeyser, M., Verfaillie, K., \& Vanrie, J. (2002). Creating stimuli for the study of biological-motion perception. Behavior Research Methods, Instruments, E Computers: A Journal of the Psychonomic Society, Inc., 34(3), 375-382. 
Fang, F., \& He, S. (2005). Cortical responses to invisible objects in the human dorsal and ventral pathways. Nature Neuroscience, $8(10)$ 1380-1385. doi:10.1038/nn1537.

Gottschaldt, K. (1926). Über den Einfluß der Erfahrung auf die Wahrnehmung von Figuren. I. Über den Einfluß gehäufter Einprägung von Figuren auf ihre Sichtbarkeit in umfassenden Konfigurationen [About the influence of experience on the perception of figures]. Psychologische Forschung, 8, 261-317.

Hochstein, S., \& Ahissar, M. (2002). View from the top: Hierarchies and reverse hierarchies in the visual system. Neuron, 36(5), 791-804.

Johansson, G. (1973). Visual perception of biological motion and a model for its analysis. Perception and Psychophysics, 14(2), 201-211.

Koffka, K. (1935). Principles of Gestalt psychology. New York, NY: Harcourt Brace Jovanovich.

Pavlova, M., \& Sokolov, A. (2000). Orientation specificity in biological motion perception. Perception \& Psychophysics, 62(5), 889-899.

Pelli, D. G. (1997). The VideoToolbox software for visual psychophysics: transforming numbers into movies. Spatial Vision, 10, 437-442. doi:10.1163/156856897X00366.

Rensink, R. A., O’Regan, J. K., \& Clark, J. J. (1997). To see or not to see: The need for attention to perceive changes in scenes. Psychological Science, 8(5), 368-373. doi:10.1111/j.1467-9280.1997.tb00427.x.

Restle, F. (1979). Coding theory of the perception of motion configurations. Psychological Review, 86(1), 1-24.

Saygin, A. P., Driver, J., \& de Sa, V. R. (2008). In the footsteps of biological motion and multisensory perception: judgments of audiovisual temporal relations are enhanced for upright walkers. Psychological Science: A Journal of the American Psychological Society/APS, 19(5), 469-475. doi:10.1111/j.1467-9280.2008.02111.x.
Suchow, J. W., \& Alvarez, G. A. (2011). Motion silences awareness of visual change. Current Biology, 21(2), 140-143. doi:10.1016/ j.cub.2010.12.019.

Sumi, S. (1984). Upside-down presentation of the Johansson moving light-spot pattern. Perception, 13(3), 283-286.

Tadin, D., Lappin, J. S., Blake, R., \& Grossman, E. D. (2002). What constitutes an efficient reference frame for vision? Nature Neuroscience, 5(10), 1010-1015. doi:10.1038/nn914.

Thornton, I. M., \& Vuong, Q. C. (2004). Incidental processing of biological motion. Current Biology: CB, 14(12), 1084-1089. doi:10.1016/ j.cub.2004.06.025.

Thorpe, S., Fize, D., \& Marlot, C. (1996). Speed of processing in the human visual system. Nature, 381(6582), 520-522. doi:10.1038/381520a0.

Treisman, A. (1996). The binding problem. Current Opinion in Neurobiology, 6(2), 171-178.

Treisman, A. M., \& Gelade, G. (1980). A feature-integration theory of attention. Cognitive Psychology, 12(1), 97-136.

Vanrie, J., \& Verfaillie, K. (2004). Perception of biological motion: A stimulus set of human point-light actions. Behavior Research Methods Instruments \& Computers, 36(4), 625-629.

Wilford, M. M., \& Wells, G. L. (2010). Does facial processing prioritize change detection?: change blindness illustrates costs and benefits of holistic processing. Psychological Science, 21(11), 1611-1615. doi: $10.1177 / 0956797610385952$.

Williams, M. A., Dang, S., \& Kanwisher, N. G. (2007). Only some spatial patterns of fMRI response are read out in task performance. Nature Neuroscience, 10(6), 685-686. doi:10.1038/nn1900. 